\title{
Computational Prediction of Estrogenic Micropollutants Removal from Lignin Surface Using Ionic Liquids
}

\author{
Roohollah Hafizi ${ }^{1}$, Mohammad Ali Amani ${ }^{2}$, Ramezan Ali Taheri ${ }^{3}$ \\ ${ }^{1}$ Department of Physics, Isfahan University of Technology, Isfahan, Iran \\ ${ }^{2}$ Applied Biotechnology Research Center, Baqiyatallah University of Medical Sciences, Tehran, Iran \\ ${ }^{3}$ Nanobiotechnology Research Center, Baqiyatallah University of Medical Sciences, Tehran, Iran
}

Corresponding Author: Mohammad Ali Amani, PhD, Applied Biotechnology Research Center, Baqiyatallah University of Medical Sciences, Tehran, Iran. Email: rsr.amani@bmsu.ac.ir

Received April 22, 2019; Accepted July 3, 2019; Online Published September 1, 2019

\begin{abstract}
Introduction: Estrogens are of the most dangerous micro/nanopollutants that have shown severe influences on the ecosystems and microorganisms. There is an ever-increasing demand to reliably detect and practically remove these estrogens from effluents. In a recently proposed method, estrogens can be detected and removed from effluents using a sampler (lignin). In this study it has been shown that ionic liquids are a potential choice to separate the adsorbed estrogens from the surface of "dirty" lignin so that the sampler could be reused.

Materials and Methods: More than 300 ionic liquids were screened for removing estrogens from the lignin surface by employing a quantum chemistry method, COnductor-like Screening MOdel (COSMO), to determine the interaction quality between the ionic liquid and eight estrogens of interest.

Results: The results revealed that there are at least 24 solvents that can remove adsorbed estrogens from the surface of lignin.

Conclusions: This prediction completes the cycle of reusing lignin as an efficient polymeric sampler to remove estrogens from effluents and provokes experimental justifications.

Keywords: Estrogenic Micropollutants, Water Effluent, Computational Chemistry, lonic Liquids, Polymeric Samplers, Lignin

Citation: Hafizi R, Amani MA, Taheri RA. Computational prediction of estrogenic micropollutants removal from lignin surface using ionic liquids. J Appl Biotechnol Rep. 2019;6(3):125-128. doi:10.29252/JABR.06.03.08.
\end{abstract}

\section{Introduction}

Ecosystems have been severely affected by biologically active micropollutants such as estrogens which are inadvertently released into the environment. ${ }^{1-4}$ These micropollutants have entered the environment by human activities, and despite deleterious effects, their concentrations are at trace level (i.e., up to the microgram per liter range). Due to the low concentration and small size of these types of contaminations (a few nanometers, typically), conventional samplers of water treatment are ineffectual. ${ }^{5}$ So, finding reliable technologies to detect and remove them from water resources is an inevitable task.

Polymeric passive samplers are of the most reliable devices for practical measurement and detection of even small concentrations of micropollutants in water effluents. ${ }^{5,6}$ In the fabrication of passive polymeric samplers, the efficient selection of materials plays a crucial role as the interaction between the sampler and micropollutants is very desirable, but the interaction among sampler and water is unfavorable. The task of finding proper sampler materials remains as a challenge to empirical methods and trial and error experiments ${ }^{1}$ as a wide variety of polymers that are available and synthesized nowadays. ${ }^{78}$ Moreover, the cost of doing experiments and sometimes inaccessibility of required materials/conditions are other impetus to motivate researchers to scan a wider range of involved parameters by doing computational simulations and investigations. In the particular case of samplers, some suitable samplers have been discovered by using computational methods, recently. ${ }^{1,6}$

In a recent project, by using a theoretical approach ${ }^{3}$ to model the interaction between lignin and eight estrogens, lignin was identified as a potential material for adsorption of estrogenic micropollutants and thus fabrication of passive polymeric samplers. ${ }^{9}$ Lignin seems an applicable choice because in one hand it is one of the most widely available biomacromolecules on the earth, ${ }^{2}$ and in the other, the use of which brings sustainability and low costs in the application of samplers. However, the possibility of cleaning and reusing lignin to remove estrogens has not been addressed in the previous report. In other words, to guarantee the reusability of polymeric samplers in separation practices, the removal of adsorbed pollutants from the surface of polymeric samplers is required at the final step.

As lignin polymer is solid, the chemical cleaning process can

Copyright (C) 2019 The Author(s). This is an open-access article distributed under the terms of the Creative Commons Attribution License (http:// creativecommons.org/licenses/by/4.0), which permits unrestricted use, distribution, and reproduction in any medium, provided the original work is properly cited. 
be done by employing a solvent. While, there is a wide range of solvents available commercially, an appropriate solvent is the one which is suitable for washing the estrogens from the surface of "dirty lignin". It is also crucial that estrogens in the "dirty" solvent could be separated easily, so that the cycle of estrogen removal is completed. Due to their special characteristics, ionic liquids can be good candidates for such a purpose. If proven to be successful in adsorption of estrogens, pollutants can be separated from ionic liquids following a repetitive exposure to a varying electric field.

To assess the reliability of ionic liquids for sampler cleaning (removal of estrogens from sampler surface), the interactions between the micropollutants, sampler, and different ionic liquids need to be studied. ${ }^{6}$ In this study, the suitability of a large number of ionic liquids (more than 300 formed by 19 anions and 16 cations) for the removal of estrogens from the sampler surface was investigated using COnductor-like Screening MOdel (COSMO) sigma profiles. The details of the used methods and the obtained results are presented in the following sections.

\section{Materials and Methods}

In practices, commonly, a flat sheet polymeric sampler is immersed into the effluent where it interacts with water and micropollutants present in the effluent as shown in Figure 1. The detection and adsorption of estrogens by sampler is governed by the surface interactions between them. ${ }^{2,3}$ After contact and a successful adsorption process, the surface of sampler would be covered by adsorbed estrogens which need to be removed so that the sampler can be reusable. Based on the excellent chemical characteristics of ionic liquids, it is believed that these solvents are potential candidates to efficiently separate estrogens from the "dirty" sampler.

To examine this hypothesis, the COSMO model was used. The COSMO sigma profiles would provide a quick and reliable rout for the determination of the interactions between molecules., ${ }^{2,3}$ Sigma profile is the probability distribution of screening charge on the molecular surface with screening charge density $(\sigma)$ shown as $p(\sigma) .{ }^{10-12}$ The particles may have desirable interactions provided that they show matched Sigma profiles and almost identical peaks., ${ }^{2,3}$ Charge density of the ground state of the components is obtained by using computational quantum mechanical methods in the framework of density functional theory (DFT). This approach which asserts that the total energy of a system is a unique functional of charge density, has been very successful in the calculation of density-related properties, in a way that Walter Kohn was awarded half of the Nobel prize in chemistry 1998 for his development of the DFT. ${ }^{13}$

In the present study, the basic quantum chemical data were retrieved from Jooshani et al study ${ }^{14}$ where DFT calculations were carried out using the DMol3 package. For geometry optimization as well as energy minimization of molecules, a generalized gradient approximation functional with VolskoWilk-Nusair correlation, and a double numerical basis set including d-polarization function were employed as recommended elsewhere.,3

\section{Results}

In Figure 2, the sigma profiles are illustrated for the estrogens of interest and lignin based sampler in aqueous media. A narrow sigma profile located inside the sigma cutoff for hydrogen bonding, i.e. $-7.9 \times 10^{-3} \leq \sigma \leq 7.9 \times 10^{-3}$ e/ $\AA^{2}$ is obtained for Estrone denoting its slight hydrophilic nature. ${ }^{3}$ The hydrogens generate strong peak around $-3 \times 10^{-3}$ e/ $\AA^{2}$ which may lead to weak hydrogen bonding noting to the sigma cutoff for hydrogen bonding. The lone-pair electrons on oxygen on carboxyl group generate peak around $\sigma=1.3$ $\times 10^{-2} \mathrm{e} / \AA^{2}$. The exposed surfaces of carbon atoms caused shoulder like peak around $2.3 \times 10^{-3} \mathrm{e} / \AA^{2}$. The peak at $\sigma=5$ $\times 10^{-3} \mathrm{e} / \AA^{2}$ is due to the $\pi$-faced caused by the carbon rings.

The next step is to check the quality of interaction between ionic liquids and adsorbed estrogens on lignin. The obtained sigma profiles of all 304 ionic liquids were calculated using the procedure explained in the previous section and are reported in the supplementary information. As mentioned

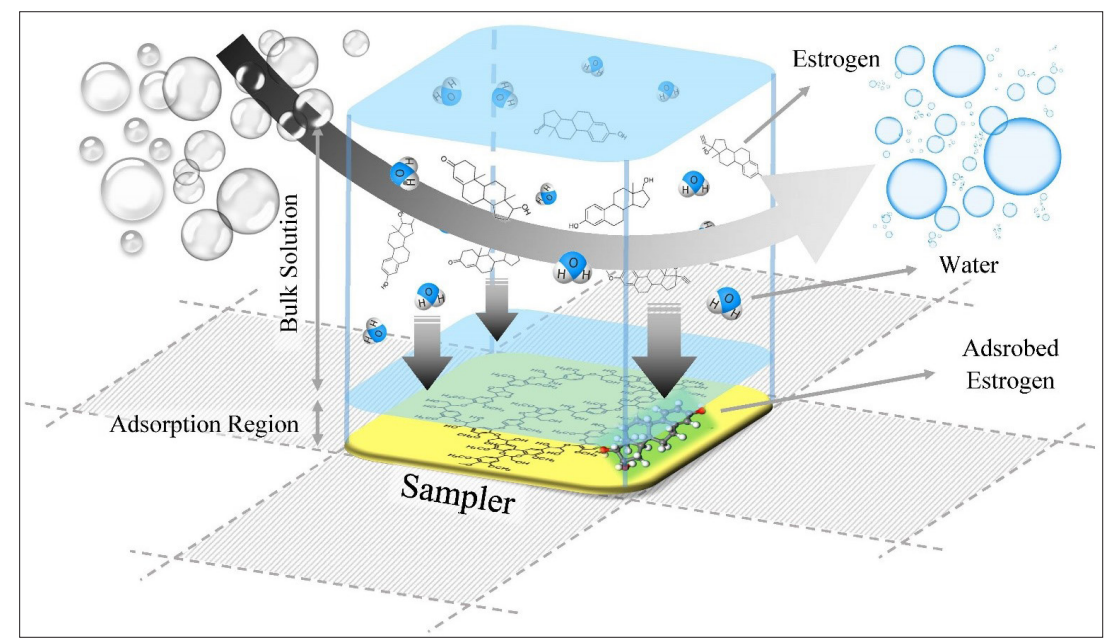

Figure 1. Schematic of the Estrogenic Containment Uptake Using Polymeric Passive Samplers. The yellow surface represents a small part of the polymer sampler. In a polluted effluent (bulk solution), the estrogenic micropollutants in aqueous phase, upon approaching enough to the sampler surface i.e. inside 'adsorption region', would be absorbed if their sigma profiles match to that of sampler's material. 


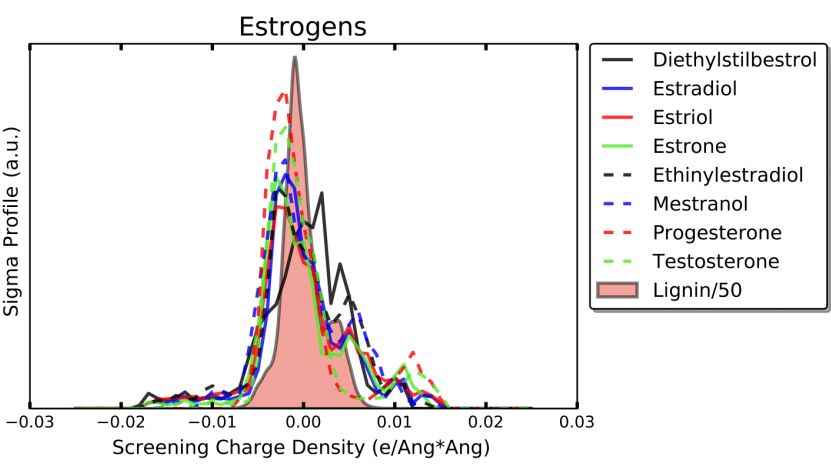

Figure 2. Obtained Sigma Profiles of Investigated Estrogens and Lignin Based Sampler in an Aqueous Media.

earlier, the probability of interaction between a specific ionic liquid and an estrogen is directly proportional to the extent of match (overlap) between their sigma profiles. ${ }^{3}$ Thus, in order to check the possibility of removing adsorbed estrogens from the surface of lignin, the matching sigma profile was checked by comparing the major peaks and overlap area in the sigma profile of ionic liquids (all possible combinations of investigated anion-cation pairs), lignin and those of estrogenic micro pollutants.

Taking a comparative approach, ionic liquids were classified by the amount of matching found in sigma profiles of the ionic liquid, lignin, and the estrogen. Based on the comparison, the ionic liquids were put in 10 classes, where 10 corresponds to the best choice of anion-cation pairs, and zero refers to the worst case where there is negligible or no overlap in sigma profiles. The corresponding results are shown in the heat maps of Figure 3. In this figure, the values corresponding to different anion-cation pairs are represented as colors. The better the match between the anion-cation pair and estrogen, the higher rank it gets, and the darker square it has in the heat maps; the color bar on the right-hand side of each heat map shows the color corresponding to each value. In other words, larger values (higher ranks) are represented by small dark blue pixels and smaller values (lower ranks or poorer matches) by lighter squares. So, to identify potential ionic liquids that are suitable for removing all eight estrogens from the surface of the "dirty" lignin, we need to look for dark squares which are common in all eight heat maps of Figure 3.

\section{Discussion}

Based on the results of sigma profiles (Figure 2), for Estradiol and Estriol, the strong peak around $-3 \times 10^{-3} \mathrm{e} / \AA^{2}$ is associated to the hydrogens and peak at $\sigma=5 \times 10^{-3} \mathrm{e} / \AA^{2}$ is due to the $\pi$-faced caused by the carbon rings. In the case of Estriol, the $-\mathrm{OH}$ group attached to five-member carbon ring generates peak around $6 \times 10^{-3} \mathrm{e} / \AA^{2}$. Moreover, the peak at $\sigma=5 \times 10^{-3} \mathrm{e} /$ $\AA^{2}$ is wider in Estriol because of the addition of a $-\mathrm{CH}$ group attached to the five-member carbon rings by a triple bond on the same carbon atom as the $-\mathrm{OH}$ group attached. For the next estrogen, ethinylestradiol, the strong peak around $-3 \times 10^{-3} \mathrm{e} / \AA^{2}$ is associated to the hydrogens; in contrast, for Diethylstilbestrol, the two $-\mathrm{CH}_{3}$ groups generate two peaks around $0-2 \times 10^{-3} \mathrm{e} / \AA^{2}$. The combination of exposed surfaces
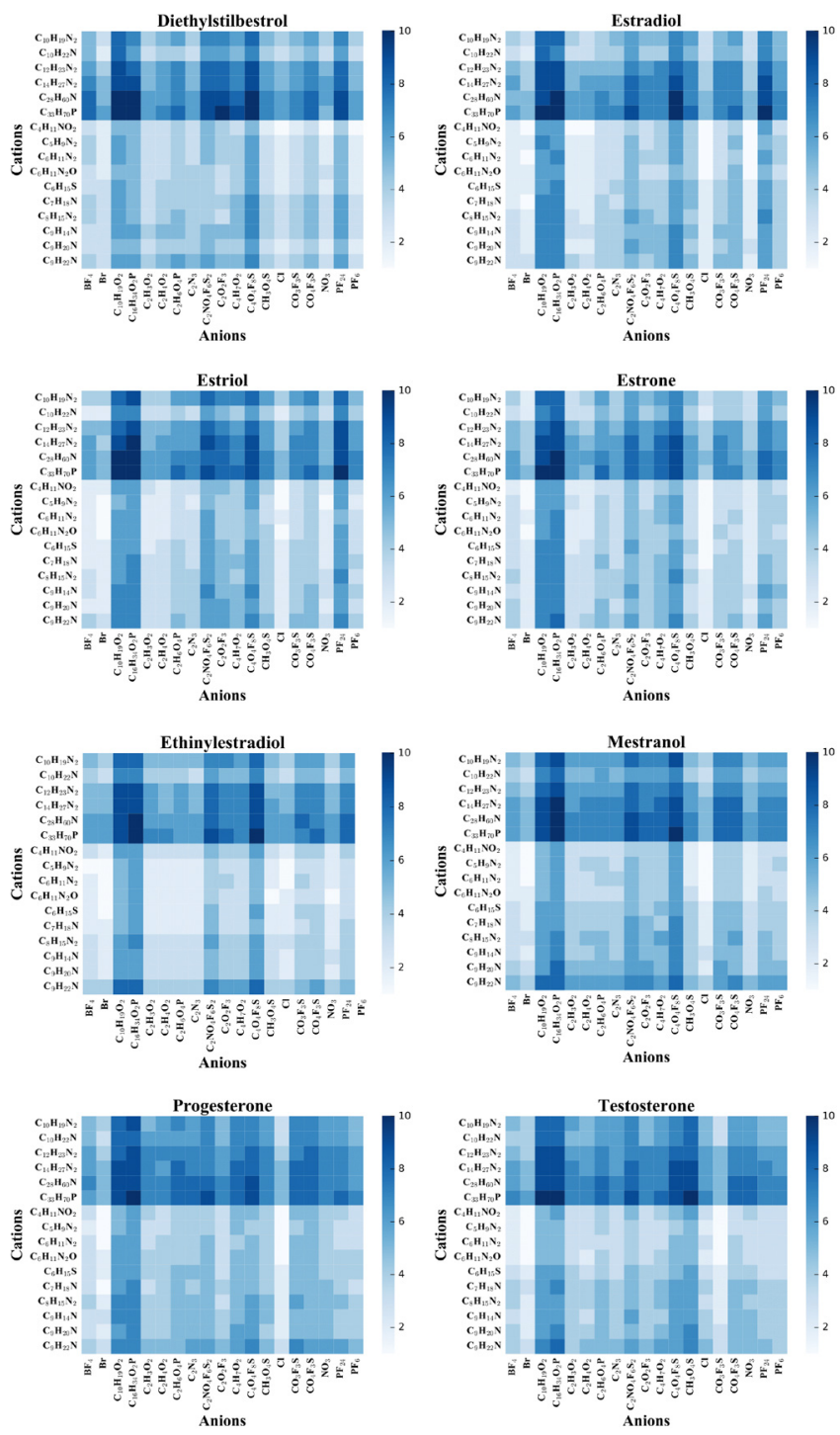

Figure 3. Mapping of Potential Ionic Liquids in Estrogenic Micropollutants Uptake. Horizontal and vertical axes show the anion and cation part of the ionic liquids, respectively. Darker colors correspond to more successful combinations of anion-cation in estrogenic micropollutants removal.

of carbon atoms and the $\pi$-faced caused by the carbon rings, generate peak around $3.5 \times 10^{-3} \mathrm{e} / \AA^{2}$. The two polar hydrogen atoms in $-\mathrm{OH}$ generate peaks around $\sigma=-1.65 \times 10^{-2}$ to 1.4 $\times 10^{-3} \mathrm{e} / \AA^{2}$. Mestranol has a strong peak around $-3 \times 10^{-3} \mathrm{e} / \AA^{2}$ which is associated to the hydrogens. The peak at $\sigma=5 \times 10^{-3}$ $\mathrm{e} / \AA^{2}$ is due to the $\pi$-faced caused by the carbon rings, while the lone-pair electrons on oxygen generates peak around $\sigma=$ $1.3 \times 10^{-2} \mathrm{e} / \AA^{2}$. For Testosterone, the observed peak around $\sigma$ $=1.3 \times 10^{-2} \mathrm{e} / \AA^{2}$ is due to the lone-pair electrons on oxygen. On the other hand, the hydrogens in - $\mathrm{OH}$ group generate a strong peak around $-1.3 \times 10^{-2} \mathrm{e} / \AA^{2}$. Finally, the strong peak around $-3 \times 10^{-3} \mathrm{e} / \AA^{2}$ is related to the hydrogens which are polarized due to the presence of oxygen atom; this peak was also observed for progesterone. The shoulder-like peak around $3.5 \times 10^{-3} \mathrm{e} / \AA^{2}$ obtained in sigma profile for lignin is due to the combination of exposed surfaces of carbon atoms and the $\pi$-faced caused by the carbon rings. The polarized 
hydrogens due to the presence of oxygen caused strong peak around $-2 \times 10^{-3} \mathrm{e} / \AA^{2}$. The lone-pair electrons on oxygen generated peak around $\sigma=1.4 \times 10^{-2} \mathrm{e} / \AA^{2} \mathrm{e}$ for water. The two polar hydrogen atoms, also, generated a peak at $\sigma=-1.4 \times 10^{-}$ ${ }^{2} \mathrm{e} / \AA^{2}{ }^{2,}{ }^{9}$ These observations read that desirable matching is evidenced; and these micro pollutants will find the lignin and its lateral surface a more relax and comfortable place to attach and interact resulting to the accumulation of micropollutants over and around lignin. ${ }^{9}$

Concerning the choice of cations in Figure 3, it seems that the first six cations, namely $\mathrm{C}_{10} \mathrm{H}_{19} \mathrm{~N}_{2}, \mathrm{C}_{10} \mathrm{H}_{22} \mathrm{~N}, \mathrm{C}_{12} \mathrm{H}_{23} \mathrm{~N}_{2}$, $\mathrm{C}_{14} \mathrm{H}_{27} \mathrm{~N}_{2}, \mathrm{C}_{28} \mathrm{H}_{60} \mathrm{~N}$, and $\mathrm{C}_{33} \mathrm{H}_{70} \mathrm{P}$, possess at least some very dark squares for all 8 estrogens of interest. On the other hand, $\mathrm{C}_{10} \mathrm{H}_{19} \mathrm{O}_{2}, \mathrm{C}_{16} \mathrm{H}_{34} \mathrm{O}_{2} \mathrm{P}, \mathrm{C}_{2} \mathrm{NO}_{4} \mathrm{~F}_{6} \mathrm{~S}_{2}$, and $\mathrm{C}_{4} \mathrm{O}_{4} \mathrm{~F}_{8} \mathrm{~S}$ are reasonable choices for the anion as they make a suitable combination with the above-mentioned cations to effectively absorb the estrogens. The ionic liquids made from these lists of cations and anions are the best choices one may expect to have. However, those which are formed from $\mathrm{C}_{28} \mathrm{H}_{60} \mathrm{~N}$, and $\mathrm{C}_{33} \mathrm{H}_{70} \mathrm{P}$ cations, and $\mathrm{C}_{10} \mathrm{H}_{19} \mathrm{O}_{2}$ and $\mathrm{C}_{16} \mathrm{H}_{34} \mathrm{O}_{2} \mathrm{P}$ anions are of particular importance. On the other hand, Figure 3 also shows that anions like bromine and chlorine are the worst choices from the anions side because they rarely form an effective combination with any of the investigated cations.

By using these ionic liquids to "clean" the "dirty" lignin, the cycle of estrogenic micropollutants removal seems completed. At first, contamination is adsorbed on the surface of lignin in water effluents. The "dirty" lignin is subsequently washed by the proposed ionic liquids, and estrogens are desorbed from its surface. As a result, lignin becomes renewed for the next estrogen uptake. The "dirty" ionic liquid might, in turn, be purified by applying a varying electric field to separate the estrogenic micro pollutants. The whole cycle is what one expects to have a reusable sampler substance for cleaning effluents from estrogens.

\section{Conclusions}

The presence of hazardous estrogenic micropollutants in water effluent instigates an inevitable need to find ways to remove them. Employing adsorption of pollutants on the surface of polymer samplers is one of the most feasible strategies to solve this issue. However, due to the practical production costs, a sampler material which is reusable is highly favored. As the second most abundant biomaterial on earth, lignin shows excellent features to be used as a polymeric sampler in up taking estrogens from aqueous media. However, the commercialization process can be more effective only if a reliable, efficient way of "cleaning" the "dirty" lignin is found. In this paper, quantum chemical simulations were used to check the suitability of 304 ionic liquids in "cleaning" lignin. The results show that there are at least 24 solvents that can remove adsorbed estrogens form lignin's surface. This prediction completes the cycle of reusing lignin as an efficient polymeric sampler and provokes experimental justifications.

Authors' Contributions

All authors equally contributed to the current study.
Conflict of Interest Disclosures

The authors declare they have no conflicts of interest.

\section{Acknowledgments}

The authors are grateful to the council of Applied Biotechnology Research Center, Baqiyatallah University of Medical Sciences for providing financial support to undertake this research.

\section{References}

1. Asgarpour Khansary M, Mellat M, Saadat SH, Fasihi-Ramandi M, Kamali M, Taheri RA. An enquiry on appropriate selection of polymers for preparation of polymeric nanosorbents and nanofiltration/ultrafiltration membranes for hormone micropollutants removal from water effluents. Chemosphere. 2017;168:91-99. doi:10.1016/j.chemosphere.2016.10.049.

2. Karezani E, Hallajisani A, Asgarpour Khansary M. A quantum mechanics/molecular mechanics (QM/MM) investigation on the mechanism of adsorptive removal of heavy metal ions by lignin: single and competitive ion adsorption. Cellulose. 2017;24(8):31313143. doi:10.1007/s10570-017-1329-3.

3. Ghasemi A, Asgarpour Khansary M, Marjani A, Shirazian S. Using quantum chemical modeling and calculations for evaluation of cellulose potential for estrogen micropollutants removal from water effluents. Chemosphere. 2017;178:411-423. doi:10.1016/j. chemosphere.2017.02.152.

4. Howarth AJ, Katz MJ, Wang TC, et al. High efficiency adsorption and removal of selenate and selenite from water using metalorganic frameworks. J Am Chem Soc. 2015;137(23):7488-7494. doi:10.1021/jacs.5b03904.

5. Asgarpour Khansary M, Shirazian S, Asadollahzadeh M. Polymerwater partition coefficients in polymeric passive samplers. Environ Sci Pollut Res Int. 2017;24(3):2627-2631. doi:10.1007/s11356016-8029-7.

6. Jooshani S, Asgarpour Khansary M, Marjani A, Shirazian S, Shang J. Contaminant uptake by polymeric passive samplers: A modeling study with experimental validation. Chem Eng Res Des. 2018;129:231-236. doi:10.1016/j.cherd.2017.11.019.

7. Asgarpour Khansary M, Aroon MA. On the consistency and correctness of thermodynamics phase equilibria modeling and correlation reports published in Fuel journal. Fuel. 2015;140:810811. doi:10.1016/j.fuel.2014.09.096.

8. Ejraei A, Shirvani S, Aroon MA, Asgarpour Khansary M, Khalaj S. Lower and upper critical solution temperatures of binary polymeric solutions. Fluid Phase Equilib. 2016;425:465-484. doi:10.1016/j. fluid.2016.06.036.

9. Hafizi R, Taheri RA, Moghimi H. Liquid phase extraction of nanosized biologically active estrogenic pollutants by using an efficient adsorbent. J Mol Liq. 2018;266:535-539. doi:10.1016/j. molliq.2018.05.079.

10. Lin ST, Sandler SI. A priori phase equilibrium prediction from a segment contribution solvation model. Ind Eng Chem Res. 2002;41(5):899-913. doi:10.1021/ie001047w.

11. Hsieh CM, Sandler SI, Lin ST. Improvements of COSMO-SAC for vapor-liquid and liquid-liquid equilibrium predictions. Fluid Phase Equilib. 2010;297(1):90-97. doi:10.1016/j.fluid.2010.06.011.

12. Klamt A. COSMO-RS from Quantum Chemistry to Fluid Phase Thermodynamics and Drug Design. Amsterdam, The Netherlands: Elsevier; 2005.

13. Jones RO. Density functional theory: Its origins, rise to prominence, and future. Rev Mod Phys. 2015;87(3):897-923. doi:10.1103/ RevModPhys.87.897.

14. Jooshani S, Khansary MA, Marjani A, Shirazian S, Shang J. Contaminant uptake by polymeric passive samplers: a modeling study with experimental validation. Chem Eng Res Des. 2018;129:231-236. doi:10.1016/j.cherd.2017.11.019. 